\title{
No such thing as a bad result
}

The failures in our experimental research are necessary steps to obtain excellent results, says Francesco Carulli.

$\mathrm{D}$ uring my undergraduate studies in materials science at the University of Milan Bicocca I developed a strong interest in renewable energy devices. Thus, when in $2013 \mathrm{I}$ had to choose a research project to complete my master's degree I decided to join the advanced photo-physics group led by Francesco Meinardi and Sergio Brovelli, who were working on solar energy. My project was on luminescent solar concentrators (LSCs), which are slabs of a transparent matrix with high diffraction index containing some sort of chromophore. The chromophores absorb a fraction of the sunlight penetrating into the slab producing and re-emitting light in the form of luminescence, which is then driven to the edge of the slab, through total internal reflection, where a photovoltaic cell is located. I was intrigued by these types of device because although they do not convert solar energy into electricity directly, they can substantially reduce the cost of photovoltaic-based devices. Furthermore, their semitransparency can facilitate the architectural integration of photovoltaic systems in buildings, for example in window panels.

The research group that I joined had just completed a study on large-area LSCs containing colloidal nanocrystals as chromophores (Nature Photon. 8, 392-399; 2014). The aim of my project was to improve the results of this study by realizing an LSC based on heavy-metalfree nanocrystals, and to take advantage of a wider fraction of solar visible light.

The focus of my project was to find a recipe to fabricate LSC devices based on a new type of quantum dot, as mentioned above. In principle, all I had to do was to extend and optimize an already tested procedure, but problems concerning the practical manufacturing of the devices emerged immediately. The manufacturing of LSCs is typically based on the dissolution of quantum dots in a monomer solution, which is then poured into a mould and subsequently thermally polymerized. The different dimensions and chemical composition of the new nanocrystal class strongly reduced its longterm solubility in the monomer solution,

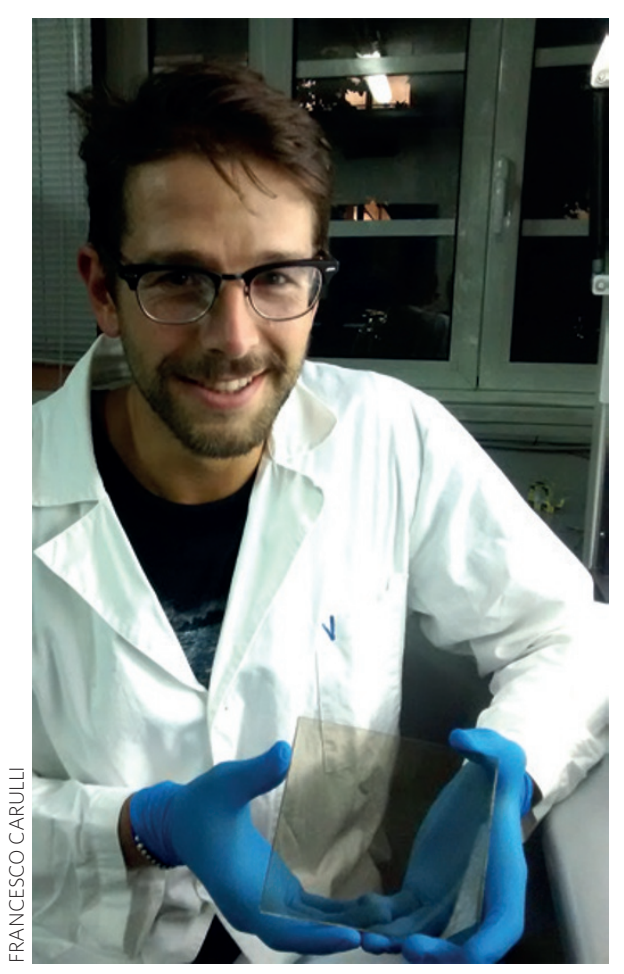

thus causing the precipitation of the nanocrystals during the polymerization process and the production of inhomogeneous devices. Moreover, there were frequent polymerization inhibition phenomena, caused by a strong interaction between the polymerization initiator and the nanocrystals.

After initially learning the synthesis and characterization techniques, I experienced a long period of setbacks related to the device manufacturing. It was really frustrating because it was clear to me what I wanted to obtain, but for six months I could not find the suitable processing method to realize it. I changed various parameters in the procedure, from the monomer type, to the initiator, to the polymerization conditions, but nothing seemed to work. It became clear to me how difficult achieving a result that in theory had seemed to be extremely simple could in fact prove.

Fortunately, my research group kept encouraging me to proceed and just one month before the end of my project, we had an idea that ended up changing our manufacturing method completely, and that led us to the publication of the results in this issue (Nature Nanotech. $10,878-885 ; 2015)$. We found a way to freeze the system in the initial stage of the process when the nanocrystals were added to the monomer solution, and during which the solubility is still acceptable. We then performed a photoactivated polymerization of the solution of monomer and quantum dots: this type of polymerization is much faster than thermal polymerization (a few minutes compared with 48 hours). During thermal polymerization the nanocrystals have enough time to precipitate, thus accumulating in the lower region of the slab. With the new recipe, based on the photopolymerization strategy, the solution of monomer and nanocrystals is set in a condition of kinetic overdrive, where nanocrystals don't have enough time to precipitate, as they remain 'frozen' inside the matrix. In this way I was able to obtain a nanocomposite material with the same optical features as the solution.

In the beginning, the time spent in the laboratory substantially increased my practical skills, even though it didn't lead to any working devices. I was therefore able to quickly optimize this new technique, producing a prototype sample and soon after the two LSCs described in this issue. The key factors to my achievement were the trust given to me by the whole group and perseverance in reaching the end goal. Without the skills developed during my period of bad results, I would have never been able to complete my master's thesis in time. This is the reason why I conserve the box of the 'notworking devices' I initially manufactured, as a reminder that the tough moments I went through were an investment in the following success.

FRANCESCO CARULLI is a PhD student in the Dipartimento di Scienza dei Materiali, University of Milan, Bicocca, Via Cozzi 55, 20125 Milano, Italy. e-mail: f.carulli@campus.unimib.it 